Rev. Inst. Flor. v. 27 n. 2 p. $145-153$ dez. 2015

http://dx.doi.org/10.4322/rif.2015.011

ISSN impresso 0103-2674/on-line 2178-5031

\title{
ESTIMATIVAS DE PARÁMETROS GENÉTICOS PARA LA SELECCIÓN DE CLONES DE EUCALYPTUS EN LA REGIÓN DE BOTUCATU, SP ${ }^{1}$
}

\section{PREDICTION OF GENETIC PARAMETERS FOR SELECTION OF CLONES OF EUCALYPTUS IN BOTUCATU, SP}

\author{
Cristiano Bueno de MORAES ${ }^{2}$; Evandro Vagner TAMBARUSSI ${ }^{3,8}$; Fernanda Maria ABÍLIO ${ }^{4}$; \\ Ana Beatriz Marques HONÓRIO²; Suzanny Héllen Viana LEAL ${ }^{2}$; Edwin Camacho PALOMINO; \\ Edmar Vinícius de CARVALHO ${ }^{2}$; Léo ZIMBACK ${ }^{6}$; Edson Seizo MORI ${ }^{7}$
}

\begin{abstract}
RESUMEN - Este trabajo trata del estudio de estimativas de parámetros genéticos cuantitativos en un ensayo clonal para la selección precoz de clones superiores de eucalipto e posterior plantío comercial. De esta forma, el objetivo de este estudio fue estimar la variabilidad genética y determinar las correlaciones genéticas entre edades de selección en un ensayo clonal en el municipio de Botucatu, SP. El experimento fue instalado en un diseño de bloques completos al azar, siendo 23 clones ( 21 tratamientos y 2 testigos), repetidas 4 veces. Las parcelas fueron formadas por 25 plantas con distanciamiento de $3,0 \times 2,0 \mathrm{~m}$. Las características silviculturales como altura de planta, diámetro a la altura del pecho - DAP y volumen de madera fueron evaluadas a los 24, 36, 48 e 60 meses después del plantío. El análisis de los parámetros genéticos fue realizado con el programa estadístico GENES. Diferencias significativas fueron observadas para todos los caracteres en el análisis. Correlaciones altas y significativas fueron observadas entre los caracteres, indicando que la selección precoz puede proporcionar ganancias genéticas de hasta $34 \%$. Los clones 18,20 e 22 presentaron las mayores estimativas para volumen de madeira, demostrando potencial para ser aprovechado en los programas de mejoramiento genético y en plantíos comerciales, proporcionando buenos niveles de productividad para la región de Botucatu, SP.
\end{abstract}

Palabras claves: mejoramiento forestal; correlación; selección juvenil.

\begin{abstract}
This work deals with the study of estimates of quantitative genetic parameters in a clonal assay for the early selection of superior clones of Eucalyptus and subsequent commercial planting. Thus, the aim of this study was to estimate the genetic variability and to determine the genetic correlations between the ages of clonal selection in a trial in the city of Botucatu, SP. The experiment was installed in a completely randomized design blocks (4) and 23 clones (21 treatment and 2 controls). The plots were formed by 25 plants with spacing of $3 \times 2 \mathrm{~m}$. Silvicultural traits as plant height, diameter at breast height - DBH and timber volume were evaluated at 24, 36, 48 and 60 months after planting. The analysis of genetic parameters was carried out with the statistical program GENES. Significant differences were observed for all traits in the analysis. High and significant correlations were observed between the traits, indicating that early selection can provide genetic gains up to $34 \%$. Clones 18, 20 and 22 had the highest volume estimates for wood, showing potential to be exploited in breeding programs and commercial plantations, providing good levels of productivity for the region of Botucatu, SP.
\end{abstract}

Keywords: tree improvement; correlation; early selection.

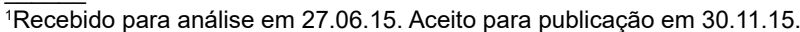

${ }^{2}$ Universidade Federal de Tocantins - UFT, Rua Padejós, L7 Chácara 69/72, Zona Rural, Caixa Postal 66, 77402-970 Gurupi, TO, Brasil.

${ }^{3}$ Universidade Estadual do Centro-Oeste, Departamento de Engenharia Florestal, PR 153 Km 7 - Riozinho, 84500-000 Irati, PR, Brasil.

${ }^{4}$ Eucatex S.A., Rua Ribeirão Preto, 811/909, Jardim Marília, 13323-010 Salto, SP, Brasil.

EUniversidade Federal do Oeste do Pará - UFOPA, Rua Vera Paz, s/n, Salé, 68035-110 Santarém, PA, Brasil.

${ }^{6}$ Instituto Florestal, Rua do Horto 931, 02377-000 São Paulo, SP, Brasil.

${ }^{7}$ Faculdade de Ciências Agronômicas de Botucatu - FCA/UNESP, Fazenda Experimental Lageado, Rua José Barbosa de Barros, 1780, 18610-307

Botucatu, SP, Brasil.

${ }^{8}$ Autor para correspondência: Evandro Vagner Tambarussi - tambarussi@gmail.com
} 


\section{INTRODUCCIÓN}

El mejoramiento genético vegetal es una de las ciencias en que el mejorador se vale de la variabilidad genética natural de las populaciones y de técnicas de selección massal o individual para formar la base del programa de mejoramiento de plantas (Zobel y Talbert, 1984).

A lo largo de 40 años de mejoramiento forestal de especies exóticas, en Brasil, se perfeccionaron procedimientos de selección de árboles e también se desenvolvieron nuevas tecnologías para mejor viabilizar el avanzo de los programas de mejoramiento, por ejemplo, para el género Eucalyptus, proporcionando expresivas ganancias de productividad en volumen de madera comparativamente a otros países (Fonseca et al., 2010).

Hoy en día, Brasil se encuentra entre los mayores productores de madera de eucalipto del mundo, siendo de 41,0 $\mathrm{m}^{3} \cdot \mathrm{ha}^{-1} \cdot \mathrm{año}^{-1}$ la media nacional de productividad con potencial para alcanzar $70 \mathrm{~m}^{3} \cdot \mathrm{ha}^{-1} \cdot$ año $^{-1}$, debido a las condiciones edafoclimaticas favorables (Associação Brasileira de Produtores de Florestas Plantadas - ABRAF, 2012).

Para mantener y aumentar la producción actual de madera, una herramienta importante que los mejoradores pueden utilizar es la técnica de la selección artificial de material genético superior con alta producción y adaptados a diversas condiciones ambientales del país, posibilitando direccionar los principales caracteres silviculturales de interés económico, por ejemplo, volumen de madera, forma del tronco, calidad de la madera y resistencia a plagas y enfermedades (Allard, 1971; Rosado et al., 2009; Madhibha et al., 2013; Miranda et al., 2013).

De esta forma, para disminuir el tiempo de selección de individuos con estos requisitos previos, la estimativa de parámetros genéticos, principalmente la correlación genética en diferentes edades, es de grande importancia en los programas de mejoramiento (Vencovsky y Barriga, 1992; Pinto et al., 2014; Moraes et al., 2014a).
En el proceso de selección precoz, los caracteres evaluados en edades jóvenes, anteriores a la de rotación, son usados como predictores en la edad final da la rotación de la cultura, anticipando las ganancias genéticas en el tempo (Weng et al., 2007; Zimback et al., 2011; Moraes et al., 2014b).

Así, el objetivo de la investigación fue estimar la variabilidad genética y determinar las correlaciones genéticas entre edades de selección en un ensayo clonal en la ciudad de Botucatu, SP.

\section{MATERIALES Y MÉTODOS}

En el año 2010, un ensayo clonal de Eucaliptus fue instalado en la Hacienda Santa Fé, de la Empresa Eucatex, localizada en el municipio de Botucatu, Estado de São Paulo. Las mudas clonales utilizadas en el experimento fueron producidas en el vivero de pesquisa de la empresa, localizado en la ciudad de Bofete/SP, siendo cinco clones de E. dunnii, cinco clones de E. saligna, cinco clones del híbrido de E. grandis x E. camaldulensis, seis clones de E. urophylla y dos testigos.

El ensayo fue instalado en un inceptisole, textura arenosa, con cobertura vegetal originaria de forestas. El clima predominante en el local es el Cfa, según la clasificación de Köppen, caracterizado como clima temperado caliente (mesotermal) húmedo, siendo la precipitación pluviométrica anual media de $1.509 \mathrm{~mm}$ y la temperatura media del mes más caliente superior a 22,0 ${ }^{\circ} \mathrm{C}$. Las coordenadas geográficas son: latitud 22050'59.16”S, longitud 48¹9'21.89”O, a una altitud de 800 metros.

El delineamiento utilizado fue el de bloques al acaso con 23 clones (21 tratamientos y dos testigos), 25 plantas/parcela (formato de cuadrado) y cuatro repeticiones. El espaciamiento entre plantas fue de $3 \times 2 \mathrm{~m}$ y borda dupla en el entorno del ensayo.

Fueron evaluadas las siguientes variables de crecimiento: 1. altura total de planta (ALT, en metros), 2. diámetro a la altura del pecho (DAP, en centímetros) y 3 . volume de madera (VOL, en $\mathrm{m}^{3}$ ) en las edades de 24, 36, 48 y 60 meses. 
Para el cálculo del volumen de madera, la expresión matemática usada fue:

$$
\operatorname{VOL}\left(\mathrm{m}^{3}\right)=\frac{\left[0,027196645 *\left(D A P^{1,752458725}\right) *\left(A L T^{1,2647048686}\right)\right]}{100}
$$

En que:

VOL: volumen del árbol en metros cúbicos;

DAP: diámetro a la altura del pecho $(1,30 \mathrm{~m}$ del suelo) en centímetros y

ALT: altura del árbol en metros.

Los análisis estadísticos, estimativas de los parámetros genéticos y correlaciones fenotípica/ genotípica fueron realizados por el programa computacional GENES (Cruz , 2006). Estimativas del coeficiente de variación genético $\left(C V_{g}=\frac{\sqrt{\sigma_{c}^{2}}}{\bar{x}} .100\right)$ donde $\sigma_{c}^{2}=$ variancia de clones y $\bar{x}=$ media de clones; heredabilidad media entre clones $\left(h_{m c}^{2}=\frac{\sigma_{c}^{2}}{\sigma_{c}^{2}+\frac{\sigma_{e}^{2}}{r}}\right)$, donde $\sigma_{e}^{2}=$ variancia del error y $r=$ número de repeticiones; relación entre el coeficiente de variación genético $\left(C V_{g} / C V_{e}\right)$; acurácia al nivel de clones $r_{\hat{a} a}=\sqrt{\hat{h}_{m c}^{2}}$ y el coeficiente de variación ambiental $\left(C V_{e}=\frac{\sqrt{Q M_{e}}}{\bar{x}} .100\right)$, donde $Q M_{e}=$ cuadrado medio del error y $\bar{x}=$ media de clones. La ganancia genetica de selección fue estimado a partir de la multiplicación de la heredabilidad por el diferencial de selección, en el que fue adoptado el criterio de $10 \%$ arriba de la media para la selección de los clones.

\section{RESULTADOS Y DISCUCIÓN}

En la región de Botucatu/SP fue evidente la heterogeneidad observada para los caracteres silviculturales evaluados DAP, ALT y VOL en términos del $C V(\%)$ para los diferentes materiales genéticos clonales de Eucalyptus (Tabla 1), pero presentando diferencias significativas entre los tratamientos (datos no mostrados), evidenciados por el análisis estadístico entre los clones evaluados.

Tabla 1. Estimativas de medias y coeficiente de variación experimental ( $C V$ exp \%) para los caracteres: diámetro a la altura del pecho - DAP, altura de plantas - ALT y volumen de madera - VOL evaluados a las edades de 24, 36, 48 y 60 meses en el ensayo clonal en Botucatu, SP.

Table 1. Estimates of mean and coefficient of the experiment variation $(C V \exp \%)$ for traits: diameter at breast height - DBH, plant height - ALT, and timber volume - VOL measured at 24, 36, 48 and 60 months for the clonal assay at Botucatu, SP.

\begin{tabular}{cccc}
\hline Edad (meses) & Caracteres & Media & CV exp (\%) \\
\hline 24 & DAP* & 3,74 & 11,09 \\
36 & & 8,13 & 7,17 \\
48 & & 10,99 & 6,96 \\
60 & & 15,99 & 4,78 \\
\hline 24 & ALT & 4,34 & 10,80 \\
36 & & 10,58 & 8,17 \\
48 & & 15,74 & 6,62 \\
60 & & 15,74 & 6,62 \\
\hline 24 & VOL & 0,0029 & 24,74 \\
36 & & 0,0310 & 12,21 \\
48 & & 0,0851 & 11,44 \\
60 & & 0,1665 & 9,61 \\
\hline
\end{tabular}

*DAP (cm); ALT (m) y VOL $\left(\mathrm{m}^{3}\right)$.

*Diameter at breast height $(\mathrm{DBH}, \mathrm{cm})$; ALT $(\mathrm{m})$ and VOL $\left(\mathrm{m}^{3}\right)$. 
Los $C V$ exp (\%) de la altura y DAP no fueron elevados, posibilitando ser posible una selección indirecta para el carácter VOL debido a la consistencia de la correlación genética. Estos valores ( $C V$ exp) variaron de 4,8 \% a 24,7\%para los caracteres DAP, altura y volumen, demostrando una buena precisión de los experimentos (Pimentel-Gomes y Garcia, 2002; Garcia y Nogueira, 2005). Valores semejantes fueron observados por Paula et al. (2002),
Moraes et al. (2014a) y Pinto et al. (2014) estudiando también especies del género Eucalyptus.

La relación entre el coeficiente de variación genética $\left(\mathrm{CV}_{g} \%\right)$ y el coeficiente de variación experimental ( $C V$ exp \%) en la Tabla 2, presento valores más altos para el carácter VOL en las edades de 36, 48 y 60 meses $\left(C V_{r}\right)$, seguida de los caracteres ALT y DAP. Cuanto mayores o iguales a uno los valores de este parámetro, se torna más fácil seleccionar el carácter (Vencovsky, 1978; Cruz et al., 2004).

Tabla 2. Estimativas del coeficiente de variación genético $\left(C V_{g} \%\right)$, heredabilidad media entre clones $\left(h_{m c}^{2} \%\right)$, relación entre el coeficiente de variación genético, acurácia al nivel de progénies $\left(\mathrm{r}_{\hat{a} a}\right)$ y el coeficiente de variación ambiental $\left(C V_{r}\right)$ para los caracteres silviculturales: diámetro a la altura del pecho - DAP, altura de plantas - ALT y volumen de madera - VOL evaluados a los 24, 36, 48 y 60 meses de edad en el ensayo clonal en Botucatu, SP.

Table 2. Estimates of coefficient of genetic variation $\left(C V_{g} \%\right)$, mean heritability between clones $\left(h_{m c}^{2} \%\right)$, relationship among the coefficient of genetic variation, accuracy at the progeny level $\left(\mathrm{r}_{\hat{a} a}\right)$, and the coefficient of environmental variation $\left(\mathrm{CV}_{r}\right)$ for silvicultural traits: diameter breast height - DBH, plant height - ALT, and timber volume - VOL at 24, 36, 48 and 60 months for the clonal assay at Botucatu, SP.

\begin{tabular}{cccccc}
\hline Edad (meses) & Caracteres & $\mathrm{CV}_{\mathrm{g}}(\%)$ & $\mathrm{h}_{\mathrm{mc}}^{2}(\%)$ & $\mathrm{r}_{\text {âa }}$ & $\mathrm{CV}_{\mathrm{r}}$ \\
\hline 24 & $\left(^{*}\right)$ DAP & 17,63 & 91 & 95 & 1,59 \\
36 & & 9,70 & 88 & 94 & 1,35 \\
48 & & 10,22 & 90 & 95 & 1,47 \\
60 & & 90 & 95 & 1,47 \\
\hline 24 & ALT & 16,36 & 90 & 95 & 1,52 \\
36 & & 12,90 & 91 & 95 & 1,58 \\
48 & & 92 & 96 & 1,70 \\
60 & & 11,24 & 92 & 96 & 1,70 \\
\hline 24 & VOL & 38,07 & 90 & 95 & 1,54 \\
36 & & 21,45 & 93 & 96 & 1,76 \\
48 & & 21,88 & 94 & 97 & 1,91 \\
60 & & 94 & 97 & 1,94 \\
\hline
\end{tabular}

*DAP (cm); ALT (m) y VOL $\left(\mathrm{m}^{3}\right)$.

*Diameter at breast height (DBH, $\mathrm{cm})$; ALT $(\mathrm{m})$ and VOL $\left(\mathrm{m}^{3}\right)$.

El coeficiente de variación genética $\left(C V_{g} \%\right)$ presento valores medios a altos para todos los caracteres y edades estudiadas. El volumen fue el que obtuvo mayor variación genética a los 60 meses $(18,7 \%)$ permitiendo la selección de los clones más productivos.

El coeficiente de heredabilidad $\left(h_{m c}^{2} \%\right)$ vario a lo largo de los años para los caracteres DAP, altura y volumen, presentando valores medios altos conforme la Tabla 2. Los coeficientes de heredabilidad media de los clones estimados a los 48 meses para el carácter volumen de madera VOL (94\%) fue superior a los demás. Entre los caracteres evaluados, el DAP fue el que presento menores coeficientes medios. A los 60 meses el valor del coeficiente de heredabilidad para VOL nuevamente fue alto (94\%), siendo un buen indicativo para la selección de materiales genéticos en edades próximas de corte para las condiciones ambientales de estudio. 
Massaro et al. (2010) estimando los parámetros genéticos de heredabilidad para dos ensayos clonales de Eucalyptus, observaron valores de 73 a 95\% en varias edades y para los mismos caracteres, demostrando que los resultados obtenidospara el ensayo clonal instalado en la región de Botucatu/SP están de acuerdo con lo que se observa en otras regiones.

Beltrame et al. (2012) evaluaron a los siete años 138 clones hídricos de eucalipto en el municipio de Tapes/RS, en las condiciones de clima subtropical húmedo y obtuvieron valores de heredabilidad para DAP de $97 \%$ y para altura $92 \%$.

El coeficiente de heredabilidad en el sentido amplio es el cuadrado de la correlación entre el valor genotípico y el valor fenotípico, utilizado para evaluación de materiales genéticos provenientes de propagación vegetativa; puede ser afectado por diversos factores, por ejemplo: el carácter estudiado, el método de estimación, la diversidad genetica en la populación, el nivel de endogamia, el tamaño de la muestra y la precisión en la conducción del experimento (White et al., 2007; Pires et al., 2011). Los estudios reportados repiten la propensión de heredabilidad alta en ensayos clonales con varias especies e híbridos de Eucalyptus; indicando que existe alta variabilidad genetica entre los clones de Botucatu/SP para realizar selección y los coeficientes de variación experimental indican buena precisión experimental.

La acurácia selectiva vario de 94 a 97\%. Eso indica una buena relación entre el valor genético verdadero y el estimado. Estos valores son compatibles con varios otros encontrados en la literatura (Moraes et al., 2014b; Reis et al., 2014) y también indican precisión experimental alta, conforme valores reportados por Resende (2002) y Resende y Duarte (2007). Las correlaciones genéticas y fenotípicas entre las diferentes edades presentaron valores entre moderados y altos (Tabla 3 ). Las correlaciones fenotípicas entre las edades 24 meses (más precoz), 60 meses (próxima del final da rotación) presentaron valores propicios $(56 \%)$ para la selección con base en el DAP. Una buena alternativa para realizar selección seria entre la edad juvenil y adulta de 36 y 60 meses (66\%), en la cual parte de los individuos seleccionados a los 36 meses seria aprovechada a los 60 meses.

Tabla 3. Correlaciones genéticas $\left(r_{g}\right)$ debajo de la diagonal y fenotípicas $\left(r_{f}\right)$, encima de la diagonal, entre las edades 24, 36, 48 y 60 meses para los caracteres diámetro a la altura del pecho - DAP, altura de plantas - ALT y volumen de madera - VOL en el ensayo clonal en Botucatu, SP.

Table 3. Genetic correlations $\left(r_{g}\right)$ (below the diagonal) and phenotypic correlation $\left(r_{f}\right)$ (above the diagonal) at 24, 36, 48 and 60 months for diameter at breast height - DBH, plant height - ALT, and timber volume - VOL for the clonal assay at Botucatu, SP.

\begin{tabular}{|c|c|c|c|c|c|c|c|c|c|c|c|c|c|c|}
\hline \multirow{3}{*}{\multicolumn{2}{|c|}{$\begin{array}{c}\text { Edad } \\
\text { (Meses) }\end{array}$}} & \multirow{3}{*}{ Caracteres } & \multicolumn{12}{|c|}{$\mathrm{r}_{f}$} \\
\hline & & & \multicolumn{4}{|c|}{ DAP } & \multicolumn{4}{|c|}{ ALT } & \multicolumn{4}{|c|}{ VOL } \\
\hline & & & 24 & 36 & 48 & 60 & 24 & 36 & 48 & 60 & 24 & 36 & 48 & 60 \\
\hline \multirow{12}{*}{$\mathrm{r}_{g}$} & 24 & DAP* & - & 0,81 & 0,50 & 0,50 & 0,91 & 0,83 & 0,58 & 0,58 & 0,94 & 0,83 & 0,53 & 0,56 \\
\hline & 36 & & 0,80 & - & 0,64 & 0,64 & 0,76 & 0,92 & 0,61 & 0,61 & 0,74 & 0,92 & 0,64 & 0,66 \\
\hline & 48 & & 0,75 & 1,00 & - & 1,00 & 0,49 & 0,61 & 0,88 & 0,88 & 0,53 & 0,69 & 0,91 & 0,93 \\
\hline & 60 & & 0,75 & 1,00 & 1,00 & - & 0,49 & 0,61 & 0,88 & 0,88 & 0,53 & 0,69 & 0,91 & 0,93 \\
\hline & 24 & ALT & 0,96 & 0,83 & 0,73 & 0,73 & - & 0,81 & 0,63 & 0,63 & 0,92 & 0,81 & 0,53 & 0,57 \\
\hline & 36 & & 0,87 & 0,97 & 1,00 & 1,00 & 0,93 & - & 0,66 & 0,66 & 0,76 & 0,90 & 0,63 & 0,65 \\
\hline & 48 & & 0,88 & 1,00 & 0,89 & 0,89 & 0,90 & 1,00 & - & 1,00 & 0,60 & 0,66 & 0,82 & 0,88 \\
\hline & 60 & & 0,88 & 1,00 & 0,89 & 0,89 & 0,90 & 1,00 & 1,00 & - & 0,60 & 0,66 & 0,82 & 0,88 \\
\hline & 24 & VOL & 0,98 & 0,81 & 0,76 & 0,76 & 0,95 & 0,87 & 0,85 & 0,85 & - & 0,84 & 0,58 & 0,60 \\
\hline & 36 & & 0,82 & 0,97 & 1,00 & 1,00 & 0,85 & 0,95 & 0,96 & 0,96 & 0,85 & - & 0,76 & 0,76 \\
\hline & 48 & & 0,73 & 0,97 & 0,98 & 0,98 & 0,72 & 0,93 & 0,88 & 0,88 & 0,78 & 1,00 & - & 0,99 \\
\hline & 60 & & 0,77 & 1,00 & 0,98 & 0,98 & 0,76 & 0,97 & 0,92 & 0,92 & 0,81 & 1,00 & 1,00 & - \\
\hline
\end{tabular}

$\mathrm{p}<0,01$ (todos los valores presentaron efecto significativo por el test $\mathrm{t}$ ).

*DAP (cm); ALT (m) y VOL $\left(\mathrm{m}^{3}\right)$.

$\mathrm{p}<0.01$ (all values are significant according to the t-test).

*Diameter at breast height $(\mathrm{DBH}, \mathrm{cm})$; ALT $(\mathrm{m})$ and VOL $\left(\mathrm{m}^{3}\right)$. 
Cuanto a las correlaciones genéticas para DAP, los valores variaron de 75 a $100 \%$ para las edades de 48 y 60 meses. A pesar del valor de la correlación genética entre las edades de 24 y 60 meses se presente moderado (75\%), se muestra todavía atrayente para la selección, delante de la posibilidad de disminuir el tiempo para atender el programa de mejoramiento genético (Kageyama y Vencovsky, 1983). Massaro et al. (2010) consiguieron correlación genética de $87 \%$ entre 25 y 72 meses para DAP, siendo superior al obtenido en este trabajo a los 24 y 60 meses.

Para ALT, la mayor correlación genética (87\%) fue entre las edades de 36 y 60 meses y la menor $(73 \%)$ fue entre 24 y 60 meses; y en el trabajo de Massaro et al. (2010) fue obtenido un valor superior $(87 \%)$ entre 24 y 72 meses. La mayor correlación fenotípica fue entre las edades de 48 y 60 meses (86\%). Las correlaciones fenotípicas entre la edad más juvenil (24 meses) y adulta (60 meses) fueron relativamente bajas para ALT (49\%), diferente de $(70 \%)$ encontrados por Moraes et al. (2014a).

Evidentemente, el carácter que mejor representa las ganancias comerciales es el volumen de madera (VOL). Para este carácter la mayor correlación fenotípica ocurrió entre la edad de 48 y 60 meses (99\%), puede ser usada en selección precoz en los programas de mejoramiento genético; entretanto, la correlación de 36 con 60 meses también se presenta buena para selección $(76 \%)$.
Entre las correlaciones genéticas, la mayor fue entre 36 (juvenil) y 60 meses (100\% final de rotación). Massaro et al. (2010) obtuvieron $98 \%$ entre 50 y 72 meses, siendo un valor bien aproximado, también entre $24 \mathrm{y}$ 60 meses (87\%) y 25 con 72 meses (89\%), respectivamente. Pinto et al. (2014) observaron que la selección precoz en un ensayo clonal instalado en el municipio de Guanhães/MG, para 94 clones de la especie E. urophylla mostrándose un procedimiento confiable, sin pérdida de los genotipos superiores, en comparación con la evaluación en edades más juveniles (tres años) en relación a la edad final de corte de la cultura (6 años).

En la Tabla 4 están los clones que presentaron mayor potencial para la región de Botucatu, SP, para el carácter volumen en las edades estudiadas. Los que se destacaron a los 60 meses fueron los tratamientos 13, 14, 18, 19, 20,21 e 22 , con valores $10 \%$ superiores de la media del ensayo clonal. Dos híbridos de Eucalyptus grandis vs E. camaldulensis $(13$ y 14$) \mathrm{y}$ cuatro de la especie E. urophylla $(19,20,21$ y 22$)$, revelándose como mejor clon el 20, no diferenciándose estadísticamente al $5 \%$ de probabilidad del clon 21. Reis et al. (2014) resaltan que la selección basada en la media de clones posee un mayor grado de precisión experimental que aquel basado en parcelas o en árboles individuales.

Tabla 4. Clasificación de los clones para el volumen $\left(\mathrm{m}^{3}\right)$ a los 24, 36, 48 y 60 meses en el ensayo clonal en Botucatu, SP.

Table 4. Classification of clones by volume $\left(\mathrm{m}^{3}\right)$ at 24, 36, 48 and 60 months for the clonal assay at Botucatu, SP.

\begin{tabular}{|c|c|c|c|c|c|}
\hline \multicolumn{6}{|c|}{ Volumen $\left(\mathrm{m}^{3}\right)$} \\
\hline \multirow{2}{*}{ Clones } & \multicolumn{5}{|c|}{ Meses } \\
\hline & 24 & \multicolumn{2}{|c|}{36} & 48 & 60 \\
\hline 1 & $0,0032 \mathrm{Ad}$ & 0,0289 & $\mathrm{Bc}$ & $0,0892 \mathrm{Bb}$ & $0,1784 \mathrm{Ca}$ \\
\hline 2 & $0,0028 \mathrm{Ad}$ & 0,0326 & Ac & $0,0908 \mathrm{Bb}$ & $0,1776 \mathrm{Ca}$ \\
\hline 3 & $0,0042 \mathrm{Ad}$ & 0,0355 & Ac & $0,0878 \mathrm{Bb}$ & $0,1719 \mathrm{Da}$ \\
\hline 4 & $0,0024 \mathrm{Ad}$ & 0,0284 & $\mathrm{Bc}$ & $0,0702 \mathrm{Cb}$ & $0,1457 \quad \mathrm{Ea}$ \\
\hline 5 & $0,0025 \mathrm{Ad}$ & 0,0277 & $\mathrm{Bc}$ & $0,0793 \mathrm{Bb}$ & $0,1575 \quad \mathrm{Da}$ \\
\hline
\end{tabular}


MORAES, C.B. de et al. Selección de clones de Eucalyptus

continuación - Tabla 1

continuation - Table 1

\begin{tabular}{|c|c|c|c|c|}
\hline \multicolumn{5}{|c|}{ Volumen $\left(\mathrm{m}^{3}\right)$} \\
\hline \multirow{2}{*}{ Clones } & \multicolumn{4}{|c|}{ Meses } \\
\hline & 24 & 36 & 48 & 60 \\
\hline 6 & 0,0011 Ac & $0,0144 \mathrm{Bc}$ & $0,0427 \quad \mathrm{Db}$ & $0,0916 \mathrm{Ga}$ \\
\hline 7 & $0,0016 \mathrm{Ad}$ & $0,0197 \quad \mathrm{Bc}$ & $0,0508 \mathrm{Db}$ & $0,1045 \mathrm{Ga}$ \\
\hline 8 & $0,0014 \mathrm{Ad}$ & $0,0229 \mathrm{Bc}$ & $0,0606 \mathrm{Cb}$ & $0,1257 \quad \mathrm{Fa}$ \\
\hline 9 & $0,0019 \mathrm{Ad}$ & $0,0245 \mathrm{Bc}$ & $0,0627 \mathrm{Cb}$ & $0,1302 \mathrm{Fa}$ \\
\hline 10 & $0,0035 \mathrm{Ad}$ & 0,0321 Ac & $0,0827 \mathrm{Bb}$ & $0,1636 \mathrm{Da}$ \\
\hline 11 & $0,0026 \mathrm{Ad}$ & $0,0279 \mathrm{Bc}$ & $0,0664 \mathrm{Cb}$ & $0,1417 \quad$ Ea \\
\hline 12 & $0,0027 \mathrm{Ad}$ & $0,0289 \mathrm{Bc}$ & $0,0882 \mathrm{Bb}$ & $0,1704 \mathrm{Da}$ \\
\hline 13 & $0,0047 \mathrm{Ad}$ & 0,0358 Ac & $0,0918 \mathrm{Bb}$ & $0,1815 \mathrm{Ca}$ \\
\hline 14 & $0,0043 \mathrm{Ad}$ & $0,0376 \mathrm{Ac}$ & $0,0961 \mathrm{Bb}$ & $0,1859 \mathrm{Ca}$ \\
\hline 15 & $0,0015 \mathrm{Ad}$ & $0,0291 \mathrm{Bc}$ & $0,0903 \mathrm{Bb}$ & $0,1735 \mathrm{Ca}$ \\
\hline 16 & $0,0012 \mathrm{Ad}$ & $0,0260 \mathrm{Bc}$ & $0,0835 \mathrm{Bb}$ & $0,1599 \quad \mathrm{Da}$ \\
\hline 17 & $0,0025 \mathrm{Ad}$ & 0,0357 Ac & $0,0951 \mathrm{Bb}$ & $0,1792 \mathrm{Ca}$ \\
\hline 18 & $0,0033 \mathrm{Ad}$ & 0,0397 Ac & $0,1108 \quad \mathrm{Ab}$ & $0,2066 \mathrm{Ba}$ \\
\hline 19 & $0,0035 \mathrm{Ad}$ & 0,0367 Ac & $0,0944 \mathrm{Bb}$ & $0,1824 \mathrm{Ca}$ \\
\hline 20 & $0,0050 \mathrm{Ad}$ & 0,0422 Ac & $0,1195 \mathrm{Ab}$ & 0,2212 Аа \\
\hline 21 & $0,0035 \mathrm{Ad}$ & 0,0391 Ac & $0,1139 \quad \mathrm{Ab}$ & 0,2127 Аа \\
\hline 22 & $0,0044 \mathrm{Ad}$ & 0,0388 Ac & $0,1037 \quad \mathrm{Ab}$ & $0,1965 \mathrm{Ba}$ \\
\hline 23 & $0,0023 \mathrm{Ad}$ & $0,0287 \quad \mathrm{Bc}$ & $0,0876 \mathrm{Bb}$ & $0,1702 \mathrm{Da}$ \\
\hline GS $10 \%$ & $0,00098 \mathrm{~m}^{3}$ & $0,00642 \mathrm{~m}^{3}$ & $0,01847 \mathrm{~m}^{3}$ & $0,03584 \mathrm{~m}^{3}$ \\
\hline GS $10 \%$ & $34 \%$ & $21 \%$ & $22 \%$ & $22 \%$ \\
\hline CV $(\%)$ & 13,25 & & & \\
\hline$p(\mathrm{C} \times \mathrm{A})$ & 0,0000 & & & \\
\hline
\end{tabular}

$\mathrm{GS}=$ gano genético de selección de clones que presentaron volumen $10 \%$ a mas que la media.

$\mathrm{GS}=$ genetic gains based on the selection of clones with a volume $10 \%$ greater than average.

Con relación a la ganancia genética con la selección, adoptando el criterio de escoger los clones que presentaron volúmenes $10 \%$ a mas que la media general, a los 24 meses fue obtenida la mayor estimativa (34\%) y en las edades siguientes las estimativas presentaron valores próximos $(21 \%$ a $22 \%)$.

\section{CONCLUSIONES}

a) Se observaron variaciones genéticas significativas entre los clones para los caracteres silviculturales evaluados. b) Correlaciones genéticas y fenotípicas se presentaron altas y significativas entre los caracteres observados, siendo propicio la utilización de la selección precoz a los 36 meses de edad.

c) Los clones 18, 20, 21 y 22 presentaron volúmenes de madera adecuados a los 60 meses, mostrando potencial para ser aprovechados por parte de la empresa para la región de Botucatu, SP, desde que bien aplicadas las técnicas silviculturales.

d) Las ganancias genéticas se mostraron promisores en el orden de $22 \%$ para las edades de 48 y 60 meses de edad. 


\section{AGRADECIMIENTOS}

A la empresa Eucatex por el financiamiento, conducción del experimento y por la disponibilidad de los resultados para su publicación.

\section{BIBLIOGRAFÍA}

ALLARD, R.W. Princípios de melhoramento genético de plantas. São Paulo: Edgar Blucher, 1971. $381 \mathrm{p}$.

ASSOCIAÇÃO BRASILEIRA DE PRODUTORES DE FLORESTAS PLANTADAS - ABRAF. Anuário estatístico da ABRAF 2012 - ano base 2011. Brasília, DF, 2012. 150 p.

BELTRAME, R. et al. Desempenho silvicultural e seleção precoce de clones de híbridos de eucalipto. Pesquisa Agropecuária Brasileira, v. 47, n. 6, p. 791-796, 2012.

CRUZ, C.D. Programa GENES: biometria. Viçosa, MG: UFV, 2006. 382 p.

; REGAZZI, A.J.; CARNEIRO, P.C.S. Modelos biométricos aplicados ao melhoramento genético. Viçosa, MG: UFV, 2004. v. 1, 480 p.

FONSECA, S.M. et al. Manual prático de melhoramento genético do eucalipto. Viçosa, MG: UFV, 2010. 200 p.

.; NOGUEIRA, M.C.S. Utilização da metodologia REML/BLUP na seleção de clones de eucalipto. Scientia Forestalis, v. 68, p. 107-112, 2005.

KAGEYAMA, P.Y.; VENCOVSKY, R. Variação genética em progênies de uma população de Eucalyptus grandis (Hill) Maiden. IPEF, v. 24, p. 9-26, 1983.

MADHIBHA, T. et al. Genetic parameter estimates for interspecific Eucalyptus hybrids and implications for hybrid breeding strategy. New Forests, v. 44, p. 63-84, 2013.

MASSARO, R.A.M. et al. Viabilidade de aplicação da seleção precoce em testes clonais de Eucalyptus spp. Ciência Florestal, v. 20, p. 597-609, 2010.
MIRANDA, A.C. et al. Heritability for resistance to Puccinia psidii Winter rust in Eucalyptus grandis Hill ex Maiden in Southwestern Brazil. Tree Genetics \& Genomes, v. 9, p. 1-9, 2013.

MORAES, C.B. et al. Estimativas de parâmetros genéticos para seleção precoce de clones de Eucalyptus para região com ocorrência de geadas.

Scientia Forestalis, v. 42, n. 102, 2014a.

MORAES, C.B. et al. Estimativas dos parâmetros genéticos para seleção de árvores de Eucalyptus. Scientia Forestalis, v. 42, n. 104, 2014b.

PAULA, R.C. et al. Predição de ganhos genéticos em melhoramento florestal. Pesquisa Agropecuária Brasileira, v. 37, p. 159-165, 2002.

PIMENTEL-GOMES, F.; GARCIA, C.H. Estatística aplicada a experimentos agronômicos e florestais: exposição com exemplos e orientações para uso de aplicativos. Piracicaba: FEALQ, 2002. 309 p.

PINTO, C.S. et al. Genetic variability in progenies of Eucalyptus dunnii Maiden for resistance to Puccinia psidii. Crop Breeding and Applied Biotechnology, n. 14, p. 187-193, 2014.

PIRES, I.E. et al. Genética florestal. Viçosa, MG: Arka, 2011. 318 p.

REIS, C.A.F.; SANTOS, P.E.T; PALUDZYSZYN FILHO, E. Avaliação de clones de eucalipto em Ponta Porã, Mato Grosso do Sul. Pesquisa Florestal Brasileira, v, 34, n. 80, p. 263-269, 2014.

RESENDE, M.D.V. Genética biométrica e estatística no melhoramento de plantas perenes. Brasília, DF: Embrapa Informação Tecnológica; Colombo: Embrapa Florestas, 2002. 975 p.

; DUARTE, J.B. Precisão e controle de qualidade em experimentos de avaliação de cultivares. Pesquisa Agropecuária Tropical, v. 37, n. 3, p. 182-194, 2007.

ROSADO, A.M. et al. Ganhos genéticos preditos por diferentes métodos de seleção em progênies de Eucalyptus urophylla. Pesquisa Agropecuária Brasileira, v. 44, n. 12, p. 1653-1659, 2009. 
MORAES, C.B. de et al. Selección de clones de Eucalyptus

VENCOVSKY, R. Herança quantitativa. In: PATERNIANI, E. (Coord.). Melhoramento e produção de milho no Brasil. Piracicaba: Fundação Cargill, 1978. p. 122-210.

; BARRIGA, P. Genética biométrica no fitomelhoramento. Ribeirão Preto: Sociedade Brasileira de Genética, 1992. 496 p.

WENG, Y.H. et al. Age-related trends in genetic parameters for jack pine and their implications for early selection. Silvae Genetica, v. 56, n. 5, p. 242-252, 2007.

WHITE, T.L.; ADAMS, W.T.; NEALE, D.B. Forest genetics. Oxford: $\mathrm{CAB}$ Internacional, 2007. 683 p.

ZIMBACK, L. et al. Correlações entre caracteres silviculturais durante o crescimento de Eucalyptus grandis Hill ex Maiden. Revista do Institulo Florestal, v. 23, n. 1, p. 57-67, 2011.

ZOBEL, B.; TALBERT, J. Applied forest tree improvement. New York: John Wiley \& Sons. 1984. 505 p. 